\title{
Miranda
}

Revue pluridisciplinaire du monde anglophone /

Multidisciplinary peer-reviewed journal on the English-

speaking world

16 | 2018

L'expérimental dans la littérature et les arts

contemporains

\section{Céline Murillo, Le cinéma de Jim Jarmusch}

\section{Vincent Souladié}

\section{OpenEdition}

\section{Journals}

Édition électronique

URL : http://journals.openedition.org/miranda/11610

DOI : 10.4000/miranda. 11610

ISSN : 2108-6559

\section{Éditeur}

Université Toulouse - Jean Jaurès

\section{Référence électronique}

Vincent Souladié, « Céline Murillo, Le cinéma de Jim Jarmusch », Miranda [En ligne], 16 | 2018, mis en ligne le 05 juin 2018, consulté le 16 février 2021. URL : http://journals.openedition.org/miranda/11610 ; DOI : https://doi.org/10.4000/miranda.11610

Ce document a été généré automatiquement le 16 février 2021.

\section{(†)}

Miranda is licensed under a Creative Commons Attribution-NonCommercial-NoDerivatives 4.0 International License. 


\title{
Céline Murillo, Le cinéma de Jim Jarmusch
}

\author{
Vincent Souladié
}

\section{RÉFÉRENCE}

Céline Murillo, Le cinéma de Jim Jarmusch, un monde plus loin (Paris : L'Harmattan, 2016), 300 p, ISBN 9782-2-343-09678-0

1 À ce jour, la constellation des réalisateurs américains indépendants découverts dans les années 1980 et 1990 n'a pas encore été complètement explorée par la recherche française en études cinématographiques. P.T.Anderson, Steven Soderbergh ou Richard Linklater, par exemple, attendent encore que la totalité de leur œuvre soit balisée avec rigueur. Le cinéma de Jim Jarmusch, un monde plus loin se présente comme un ouvrage important en la matière puisqu'il compense splendidement la réserve avec laquelle a trop été longtemps appréhendée l'œuvre de cet auteur. Le travail au long cours mené par Céline Murillo ne consiste pourtant pas en une révision de jugement, Jarmusch demeurant l'une des figures les plus identifiables et les plus admirées par les critiques de son époque. Le vœu exaucé par cet essai analytique est en fait celui d'un travail de recherche érudit et complet, nous invitant à revoir d'un regard mieux armé un cinéaste aimé.

2 Passée l'ère mythifiée du new Hollywood, dans les cendres de laquelle Jarmusch commence sa carrière avec Permanent Vacation (1980), le reproche le plus communément adressé aux jeunes cinéastes de sa génération fut celui de l'étoilement référentiel de leurs œuvres. Que les films soient plus que jamais habités par la mémoire cinéphile appauvrirait leur disposition à l'invention formelle, diluerait leur identité dans réseau citationnel, instiguerait la distance ironique comme seule tonalité, complexifierait leur appropriation intime. C'est là un vice contemporain auquel a vite été accolé le nom de post-modernisme : vider les images de leur réalité, ne plus savoir privilégier qu'une référence aux œuvres passées plutôt qu'une référence au monde. 
Or, c'est justement l'argument moteur dont se saisit Céline Murillo pour analyser et défendre le cinéma de Jim Jarmusch, pourtant l'un des plus épargnés par de tels anathèmes (en comparaison de Tarantino ou de Tim Burton au même moment, par exemple). Le sous-titre de l'ouvrage, Le Cinéma de Jim Jarmusch, un monde plus loin, prend acte du régime de mise à distance référentielle de l'œuvre et annonce combien cette question centrale doit être traitée avec frontalité. Le défaut d'ancrage des films dans la réalité ne convient donc pas ici d'être minoré, et le repérer ne revient pas non plus à signaler l'impuissance créatrice de l'auteur. Souhaitant « montrer comment les films de Jarmusch font reculer la référence au monde de différentes façons » (10), Céline Murillo identifie la démarche de ce dernier à une singulière pensée formelle, c'est-à-dire à l'expression personnelle de son style. Ainsi, jusque dans la persona artistique qu'il s'est construite au dehors de ses films, les «choix stylistiques et esthétiques du réalisateur [...] semblent faire de son œuvre une authentique émanation de sa personne» (9). L'intelligence du propos de Céline Murillo consiste à prendre l'analyse esthétique comme cadre intellectuel (étudier la singularité formelle et son pourvoi d'affects) alors même qu'elle se confronte à des sujets qui pourraient tendre à le conjurer au nom d'une primauté sémiologique (l'intermédialité, la réflexivité, les genres). La méthodologie n'est donc pas à proprement parler auteuriste, au sens franco-français, puisque l'auteur en question est d'abord et finement cerné à partir des signes grâce auxquels on aurait tendance à objectiver ou à relativiser sa présence (les nœuds référentiels). Cette apparente gymnastique est rendue possible par une affirmation essentielle: l'autotélisme de l'œuvre n'est pas exempt de renvois au réel. C'est justement la virtuosité accomplie par les films de Jarmusch que de toujours nous ramener « vers le monde pour en proposer une nouvelle lecture qui devient possible grâce à un décalage » (11). N'est-ce pas là le propre de l'expérience esthétique que d'être incidemment stimulé par l'organicité formelle de l'œuvre, pour que s'opère avec elle un va-et-vient intime?

Comment se manifeste alors cette mise à distance du monde vers laquelle sa reconfiguration filmique permet de faire retour? En décelant des facteurs de « déliaison entre ce qui se passe à l'écran et le monde extérieur » (11), Céline Murillo se place distinctement sous influence deleuzienne, ce qui lui permet de questionner les enjeux esthétiques de la question en étudiant, au fil des différentes parties de son ouvrage, l'aporie des images, l'intericonicité (par citations et échos internes), la défamiliarisation des choses. L'ensemble de ces éléments est in fine reformulé en de fines notifications de tonalité, indices de la sensibilité du regard jarmuschien à laquelle les images donnent corps : « la rencontre », « l'oisiveté », « la mélancolie ».

5 La première partie de l'ouvrage traite des figures de l'errance et de l'exil, traduits par Jarmusch en d'insolites expériences physiques et optiques. Il accompagne ainsi des " marginaux contemplant le monde sans intervenir, touristes se maintenant à bonne distance de l'environnement étranger dans lequel ils voyagent, observateurs hébétés de leur propre vie » (51). Céline Murillo décrit ici l'impuissance de ce décor de surface à s'actualiser comme milieu dynamique pour les corps qu'il héberge. Le lecteur reconnaîtra dans ces "personnages comme voyants» (31) les acteurs d'une «crise de l'image-action " (Deleuze, L'image-mouvement), notamment rapportés aux figures de la ballade urbaine, et lira avec attention la description précise des formes filmiques jarmuschiennes auxquelles celle-ci donne lieu. Ce défaut d'ancrage des corps dans leur environnement se met ainsi au service d'un cinéma du spleen désinvolte, mettant en 
image un monde dans lequel des personnages poètes, par sursaut de lucidité, renâclent à s'investir. On comprend que « la vision remplace l'action, vaut pour entendement et pousse par là hors du réalisme » (51).

6 À cette mise à distance du monde par souci existentiel s'ajoute le défaut d'ancrage des images dans la diégèse. C'est là que Jim Jarmusch s'affirme en cinéaste de la reprise. Amoureux des références cinéphiles, littéraires ou musicales qui ont forgé son identité d'artiste, Jarmusch les parsème dans ses films, en un palimpseste mémoriel ludique, dynamique et parfois complexe. Dans ce tissu citationnel, «feuilletage croustillant de textes » (67), les images sont délogées de leur stricte référence à la diégèse mais elles offrent aussi parfois au personnage un cadre d'épanouissement dès lors qu'il se reconnaît et se contemple lui-même comme acteur d'une réalité déracinée, qui renvoie dans l'ailleurs imaginaire d'une culture passée. « Les œuvres citées qui font appel à des récepteurs multiples créent parfois des brouillages surprenant. Mêlant les différentes matières de l'expression, ils créent une sorte d'explosion énonciative tangible pour le spectateur » (65). Dans tous les cas, « être lecteur ou spectateur isole les personnages ou bien les protège », en somme les tient judicieusement à distance du monde. La question des genres cinématographiques n'est pas en reste pour traiter cette question, surtout lorsqu'avec Ghost Dog (1999), Jarmusch travaille littéralement «l'hybridation générique entre (au moins) le film de gangster et le film de samouraï» (69), ou lorsqu'il procède à une « distorsion des conventions du western » (104) avec Dead Man (1995).

7 L'intericonicité jarmuschienne n'est d'ailleurs pas réduite à la seule problématique de la citation. En cinéaste-poète, Jarmusch œuvre à la répétition de ses propres images au sein même des films pour composer des ballets rythmiques qui entrent subtilement en dissidence avec la fiction racontée. C'est alors à une belle étude de motifs que Céline Murillo s'attèle dans la troisième partie de son ouvrage, interrogeant l'autoréférence et les répétitions. La logique de la linéarité et de la causalité classique du cinéma est mise à mal par des modalités d'abstraction plastique (la géométrie des décors), par l'itération des mêmes images ou par les boucles sonores. Bref, Céline Murillo met ici au jour un défaut d'ancrage des images dans leur récit, Jarmusch prenant pour sujet esthétique le décalage, la différenciation, l'unique et le multiple. Le spectateur y puise tout au long des films l'expérience d'un rythme visuel et musical proprement poétique. «La propriété la plus profonde de la répétition dans les films de Jarmusch est qu'elle part de la forme pure pour aller à des notions abstraites, philosophiques, en passant à peine par la représentation» (161). Cette obsession se poursuit jusque dans l'exploitation plus critique du motif de la machine, ordonnatrice chez l'homme d'un faire répétitif auquel il doit résister.

8 Enfin, le défaut d'ancrage des individus à leur propre identité fictionnelle constitue chez Jarmusch le plus profond phénomène de déliaison. Les acteurs à l'écran trahissent une mise en crise de la représentation à partir notamment d'une caractérisation éclatée. L'idée se rencontre par exemple à travers la figure de Bill/William Blake (Johnny Depp), personnage qui est tout à la fois lui-même et un autre, homonyme, dédoublement, réincarnation du poète anglais. Céline Murillo guide avec précision le lecteur dans les méandres de ces modalités de dédoublement, au sein d'œuvres qui s'ouvrent toujours et sans fin sur elles-mêmes. En fin de compte, la recherche de la part du cinéaste d'une forme d'incomplétude filmique conduit à un défaut d'ancrage du spectateur dans le monde de la fiction. «Ne pouvant donner libre cours à la pulsion narrative du 'et alors?', le spectateur reste ouvert à tous les possibles (269)». Celui-ci 
n'est donc pas refoulé à l'orée du film, il puise au contraire dans le mystère de ce flottement référentiel la liberté d'interroger autrement sa propre réalité en retour.

Même si le film est trop récent pour avoir été intégré à l'étude de Céline Murillo, qui y trouvera peut-être sujet à prolongement pour une prochaine édition augmentée, la pertinence de toutes les remarques qu'elle soulève dans son ouvrage se vérifie impeccablement dans Paterson (2016), dernier long-métrage de Jim Jarmusch en date. $\mathrm{Au}$ fil d'une structure narrative travaillant la répétition et la variation des images, le héros éponyme (Adam Driver) incarne un poète portant le même nom que la ville qu'il habite et dans laquelle il circule sans cesse au volant de son autobus, ville qui donne aussi son nom au titre d'un long poème de William Carlos Williams, cité dans le film. C'est tout le mystère poétique ouvert par le cinéma jarmuschien tel que l'analyse Céline Murillo : comment être à la fois unique et contenir en soi-même le monde entier.

\section{INDEX}

Keywords : American cinema, Jim Jarmusch, film form, aesthetics, poetry, reference, postmodernity, world, reality, encounter, idleness, melancholy, genres, intericonicity, intertextuality, wandering, urbanity, Gilles Deleuze, movement-image, disconnection, quote, hybridization, repetition, representation, intimacy, margins

Mots-clés : cinéma américain, Jim Jarmusch, formes, esthétique, poésie, référence, postmodernité, monde, réalité, rencontre, oisiveté, mélancolie, genres, intericonicité, intertextualité, errance, urbanité, Gilles Deleuze, image-mouvement, déliaison, citation, hybridation, répétition, représentation, intimité, marges

\section{AUTEURS}

\section{VINCENT SOULADIÉ}

Maître de Conférences

Université Toulouse Jean Jaurès

vincent.souladie@univ-tlse2.fr 